Check for updates

Cite this: Chem. Commun., 2021, 57,6245

Received 18th March 2021, Accepted 19th May 2021

DOI: $10.1039 / \mathrm{d} 1 \mathrm{cc} 01464 a$

rsc.li/chemcomm

\section{A transport channel-regulated MXene membrane via organic phosphonic acids for efficient water permeation $\dagger$}

\author{
Ming $\mathrm{Yi}^{\mathrm{abc}}{ }^{\mathrm{c}}$ Frédéric Héraly, ${ }^{\mathrm{c}}$ Jian Chang, ${ }^{\mathrm{C}}$ Atefeh Khorsand Kheirabad, ${ }^{\mathrm{C}}$ \\ Jiayin Yuan, (D) ${ }^{c}$ Yan Wang ${ }^{\star a b}$ and Miao Zhang (D) *c
}

\begin{abstract}
A series of organic phosphonic acids (OPAs) were applied as multifunctional spacers to enlarge the inner space of carbide $M X e n e\left(\mathrm{Ti}_{3} \mathrm{C}_{2} \mathrm{~T}_{x}\right)$ laminates. A synergistic improvement in permeance, rejection and stability is achieved via introducing OPA to create pillared laminates. This strategy provides a universal way to regulate transport channels of MXene-based membranes.
\end{abstract}

Two-dimensional transition metal carbides, nitrides, and carbonnitrides, known as MXenes, have experienced an avalanche of research advances in diverse applications, including energy storage, electrocatalysis, and water treatment, to name a few. ${ }^{1-5}$ Among them, titanium-based MXenes $\left(\mathrm{Ti}_{3} \mathrm{C}_{2} \mathrm{~T}_{x}\right)$ are of particular interest for water purification in the postgraphene era, due to their favorable features of rich surface chemistry, excellent mechanical properties, and environment-friendly characteristics. ${ }^{6-11}$ Pristine $\mathrm{Ti}_{3} \mathrm{C}_{2} \mathrm{~T}_{x}$ membranes with selective sieving of various metal ions and dye cations have been reported by Ren et al. as the first attempt of an MXene-based separation membrane. ${ }^{12}$ Thereafter, extensive strategies have been developed to engineer MXene-based nanolaminate membranes with desirable microstructures. For instance, template and spacer strategies have been developed to improve water permeability. ${ }^{13-16}$ Despite encouraging results in water permeation, the disordered interlayer space resulting from laissez-faire assembly of MXene flakes impedes the efficient separation of different molecules, due to the unstable mass transport. Moreover, for a water-related scenario, oxidative degradation of MXene nanosheets, which imperils their stability and practical significance, is

\footnotetext{
${ }^{a}$ Key Laboratory of Material Chemistry for Energy Conversion and Storage (Huazhong University of Science and Technology), Ministry of Education, Wuhan, 430074, P. R. China.E-mail: wangyan@hust.edu.cn

${ }^{b}$ Hubei Key Laboratory of Material Chemistry and Service Failure,

School of Chemistry and Chemical Engineering, Huazhong University of Science \& Technology, Wuhan, 430074, P. R. China

${ }^{c}$ Department of Materials and Environmental Chemistry, Stockholm University, Stockholm, 10691, Sweden. E-mail: miao.zhang@mmk.su.se

$\dagger$ Electronic supplementary information (ESI) available. See DOI: 10.1039/ d1cc01464a
}

another issue that needs to be considered. ${ }^{17-19}$ Therefore, a strategy to enable both fast molecular sieving and water stability remains a challenging task.

Given the 2D nature of MXene nanosheets, the critical point to engineering high-performance membranes is meticulous control of the nanogalleries formed by the stacking of adjacent nanosheets on the nanoscale. Judicious choice of crosslinkers can not only bridge the MXene nanosheets to endow them with better stability and regulate the parameters of the nanogalleries for more precise sieving, but also arrest the oxidative issue of MXene under aqueous conditions. Herein, we reported a facial strategy by introducing a series of organic phosphonic acids (OPAs) bearing different numbers of phosphonic acid groups as spacer sequences to enlarge the inner space of an MXene laminate membrane (Fig. 1). These active phosphonic acid groups tend to combine with MXene flakes to form a covalent Ti-O-P bond via interfacial nucleophilic addition and sequential condensation reaction. ${ }^{20}$ The pillared membrane with welldefined nanochannels delivers a five-fold increase in water

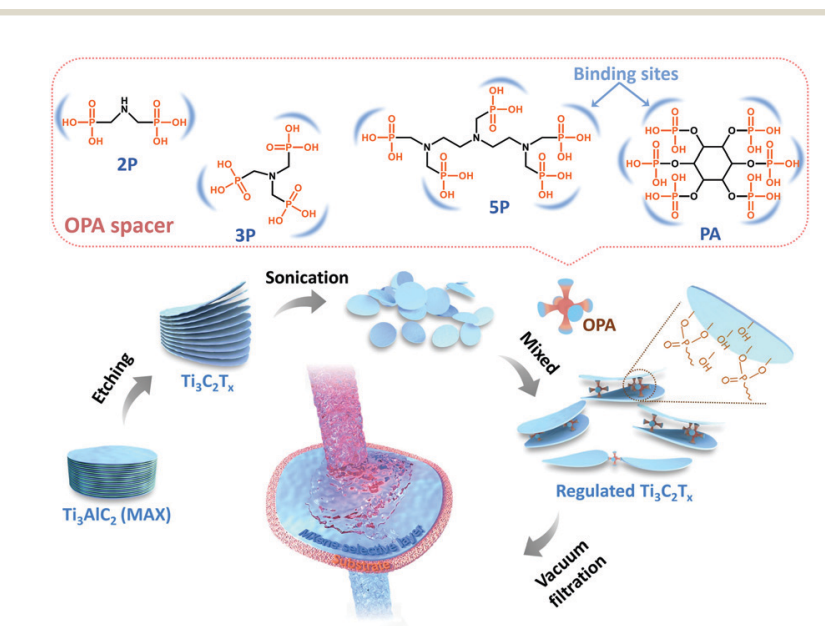

Fig. 1 Schematic of the fabrication of organic phosphonic acid (OPA) modified MXene membranes. 
permeance in comparison with the pure one. Equally importantly, the retained high rejection and the remarkable tolerance to harsh aqueous environments make this methodology a step forward for future practical applications.

The $\mathrm{Ti}_{3} \mathrm{C}_{2} \mathrm{~T}_{x}$ nanosheets were synthesized following the wellestablished procedure. ${ }^{21}$ To initiate the reaction and intercalation of OPA to $\mathrm{Ti}_{3} \mathrm{C}_{2} \mathrm{~T}_{x}$ nanosheets, certain amounts of iminodi(methylphosphonic acid), nitrilotri(methylphosphonic acid) and diethylenetriaminepentakis (methylphosphonic acid) (termed $\boldsymbol{n P}$, where $\boldsymbol{n}$ denotes the number of phosphonic acid groups), and phytic acid (PA) were added into suspensions of $\mathrm{Ti}_{3} \mathrm{C}_{2} \mathrm{~T}_{x}$. MXene membranes were prepared by vacuum filtration. The as-prepared $\mathrm{Ti}_{3} \mathrm{C}_{2} \mathrm{~T}_{x}$ nanosheets were first characterized by scanning electron microscopy (SEM, Fig. 2a), transmission electron microscopy (TEM, Fig. 2b), and atomic force microscopy (AFM, Fig. 2c). Corresponding images consistently indicate that the nanosheets are smooth yet rigid with a height of $\sim 2 \mathrm{~nm}$ and lateral size of $0.5-5 \mu \mathrm{m}$. Given the theoretical thickness of $\sim 1.0 \mathrm{~nm}$ for a monolayer, ${ }^{22}$ the extra thickness can be attributed to water molecules absorbed on the $\mathrm{Ti}_{3} \mathrm{C}_{2} \mathrm{~T}_{x}$ surface terminal groups. ${ }^{23}$ The corresponding elemental distribution of the $\mathrm{Ti}_{3} \mathrm{C}_{2} \mathrm{~T}_{x}$ nanosheets can be found in the mapping results of energy dispersive X-ray spectroscopy (EDS) (Fig. S1, ESI $\dagger$ ). Successful etching, exfoliation, and restacking are further confirmed by X-ray diffraction (XRD) patterns (Fig. S2, ESI $\dagger$ ), as the peak at $39^{\circ}$ for (104) planes disappeared and the diffraction peak for (002) planes shifted to lower angles

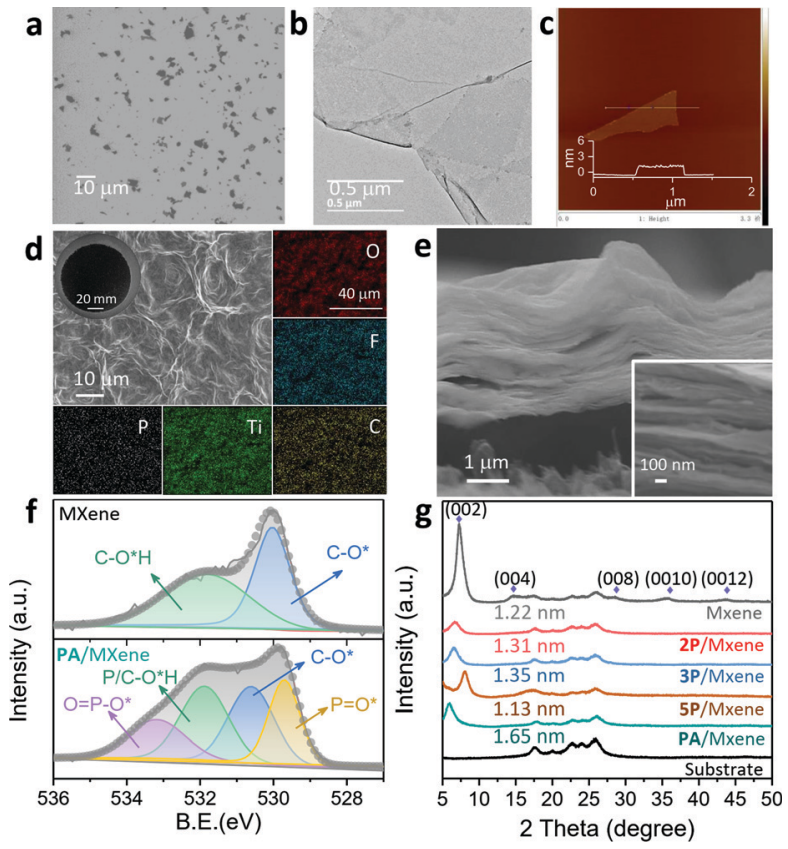

Fig. 2 (a) SEM images, (b) TEM images, and (c) AFM images of $\mathrm{Ti}_{3} \mathrm{C}_{2} \mathrm{~T}_{x}$ nanosheets. (d) Surface and the corresponding element mapping, and (e) cross-sectional SEM images of the PA/MXene membrane. Inset shows the digital photo of the corresponding membrane on a nylon- 66 substrate. (f) Peak deconvolution of narrow-scan spectra of $O 1$ s for pure and PA/MXene membranes. (g) XRD patterns of pure and OPA modified MXene membranes. after etching. ${ }^{24}$ The high-resolution transmission electron microscope (HRTEM) image identifies the high crystallinity and structural integrity of monolayer $\mathrm{Ti}_{3} \mathrm{C}_{2} \mathrm{~T}_{x}$ nanosheets (Fig. S3, ESI $\dagger$ ). Furthermore, clear lattice fringes of the Ti atom layer shown in the selected area electron diffraction (SAED) patterns indicate the well-preserved hexagonal symmetry structure (Fig. S3, ESI $\dagger$ ). ${ }^{25}$ The zeta potential of the $\mathrm{Ti}_{3} \mathrm{C}_{2} \mathrm{~T}_{x}$ suspension is measured as $\sim-38.9 \mathrm{mV}$ at $\mathrm{pH} 7$, implying a good dispersion stability of $\mathrm{Ti}_{3} \mathrm{C}_{2} \mathrm{~T}_{x}$ in water.

The dynamic light scattering (DLS) results of the corresponding mixed suspensions show that the average particle size of OPA modified $\mathrm{Ti}_{3} \mathrm{C}_{2} \mathrm{~T}_{x}$ nanosheets is enlarged compared to that of the pristine one (Fig. S4, ESI $\dagger$ ), indicating the crosslinking of MXene laminates. Accordingly, the zeta potential value of OPA-added samples is more negative than that of the pristine $\mathrm{Ti}_{3} \mathrm{C}_{2} \mathrm{~T}_{x}$ suspension, indicative of successful modification of $\mathrm{Ti}_{3} \mathrm{C}_{2} \mathrm{~T}_{x}$ nanosheets (Fig. S5, ESI $\dagger$ ). In addition, the corresponding $\mathrm{Ti}_{3} \mathrm{C}_{2} \mathrm{~T}_{x}$ nanosheets were sedimented after $12 \mathrm{~h}$ with the modification of PA (Fig. S6, ESI $\dagger$ ), further demonstrating the successful crosslinking of $\mathrm{Ti}_{3} \mathrm{C}_{2} \mathrm{~T}_{x}$ nanosheets by PA. It is worth noting that the particle size of $\mathrm{Ti}_{3} \mathrm{C}_{2} \mathrm{~T}_{x}$ nanosheets studied here follows the order of 5P/MXene $>\mathbf{2 P} /$ MXene $>$ 3P/MXene $>$ PA/MXene $>$ MXene, manifesting the differing interaction between $\mathrm{Ti}_{3} \mathrm{C}_{2} \mathrm{~T}_{x}$ nanosheets and different OPAs. This enables the possibility to produce tailor-made $\mathrm{Ti}_{3} \mathrm{C}_{2} \mathrm{~T}_{x}$ composite microstructures by varying the OPA.

Corrugations can be observed on the surface of both pristine and OPA modified MXene membranes (Fig. 2d and Fig. S7, ESI $\dagger$ ). The inset macroscopic membrane images show uniform deposition of MXene onto the support without visible defects. The OPA-modified MXene membranes display looser stacking with larger thicknesses $(2.814,2.957,3.430$ and $3.525 \mu \mathrm{m}$ for $2 \mathrm{P}, 3 \mathrm{P}, 5 \mathrm{P}$ and PA/MXene membrane, respectively) than that of the pristine one $(2.051 \mu \mathrm{m})$ (Fig. 2e and Fig. S8, ESI $\dagger$ ), which provides preliminary evidence for successful intercalation and opening of nanochannels.

Fourier transform infrared (FTIR) was applied to characterize the Ti-O-P bonding between OPA molecules and MXene nanosheets (Fig. S9, ESI $\dagger$ ). The spectrum of PA/MXene exhibited $\mathrm{P}-\mathrm{O}$ and $\mathrm{P}=\mathrm{O}$ bands at $1010 \mathrm{~cm}^{-1}, 1210 \mathrm{~cm}^{-1}$ and $1365 \mathrm{~cm}^{-1}, 20$ respectively, suggesting the successful chemical grafting of OPA molecules onto MXene nanosheets. To probe the phosphorus (P) content, X-ray photoelectron spectroscopy (XPS) analysis and EDS mapping are conducted. Unsurprisingly, all OPA modified MXene membranes possess obviously higher $\mathrm{P}$ amounts compared with the pure one (Fig. S7, S10 and Tables S1, S2, ESI $\dagger$ ). For OPA-modified MXene membranes, the peaks at 529.7 and $533.2 \mathrm{eV}$ are assigned to $\mathrm{P}=\mathrm{O}^{*}$ and $\mathrm{O}=\mathrm{P}-\mathrm{O}^{*}$ species, respectively, indicative of the presence of phosphoric acid groups (Fig. $2 \mathrm{f}$ and Fig. S10, ESI $\dagger$ ). ${ }^{26}$ These results further manifest the intercalation of OPA molecules. The cross-sectional EDS mapping indicates the homogeneous modification of OPA molecules on MXene nanosheets (Fig. S11, ESI $\dagger$ ). The direct result of the successful intercalation of OPA into nanosheets is the regulation of the interlayer distance for MXene-based membranes. This was further studied by XRD. The pure MXene membrane displays the (002) 
peak located at $7.2^{\circ},{ }^{27}$ corresponding to a $d$-spacing of $1.22 \mathrm{~nm}$ (Fig. 2g), while 2P/MXene, 3P/MXene and PA/MXene membranes show enlarged nanochannels with sizes of 1.31, 1.35 and $1.65 \mathrm{~nm}$, respectively. Such wide galleries will enable the rapid passage of water molecules through the OPA modified MXene membranes. Notably, the increase in interlayer distance is highly dependent on the size of OPA molecules. Abnormally, the 5P/MXene membrane displays a decreased nanochannel size of $1.13 \mathrm{~nm}$, resulting from the rapid sedimentation of $5 \mathbf{P}$-modified $\mathrm{Ti}_{3} \mathrm{C}_{2} \mathrm{~T}_{x}$ nanosheets before the membrane fabrication (Fig. S6, ESI $\dagger$ ). This unnatural tendency may be associated with the more flexible molecular structure of $\mathbf{5 P}$.

To showcase the practical separation performance, permeances and rejections of the membranes were measured. The loading of $\mathrm{Ti}_{3} \mathrm{C}_{2} \mathrm{~T}_{x}$ nanosheets was firstly optimized by treating with Congo Red (CR, MW of $696.66 \mathrm{~g} \mathrm{~mol}^{-1}$ ) aqueous solution (Fig. S12, ESI $\dagger$ ). The membranes with a higher loading of $\mathrm{Ti}_{3} \mathrm{C}_{2} \mathrm{~T}_{x}$ nanosheets give higher rejection and lower permeance due to there being fewer defects and larger transmission resistance, in agreement with the results on graphene and other $2 \mathrm{D}$ material-based membranes. ${ }^{28} \mathrm{~A}$ rejection of higher than $95 \%$ for CR can be achieved by pure MXene membranes with loading above $240 \mathrm{mg} \mathrm{m}^{-2}$. Given the trade-off between permeance and rejection, the MXene membranes with a loading of $240 \mathrm{mg} \mathrm{m}^{-2}$ were chosen as the benchmark for subsequent separation tests.

The proportion of OPA molecules to $\mathrm{Ti}_{3} \mathrm{C}_{2} \mathrm{~T}_{x}$ nanosheets was vital for their assembly and the channel regularity of the MXene membrane. The concentration of OPA and its reaction time with $\mathrm{Ti}_{3} \mathrm{C}_{2} \mathrm{~T}_{x}$ nanosheets were systematically optimized. Here the PA was selected as a model for the optimization. The MXene membranes show at least a two-fold increase in water flux with the addition of PA (Fig. 3a), implying the importance of the nanochannel opening to the unimpeded entrance of water into interlamination. Accordingly, the rejections increase from $96 \%$ to $99 \%$, demonstrating that the reduced defects derived from PA molecules induced pre-assembly. However, with the concentration of PA increased above $2.0 \mathrm{wt} \%$, the rejection of the PA/MXene membrane decreased sharply with a significant increase of water flux. This could be ascribed to the severe aggregation and sedimentation of $\mathrm{Ti}_{3} \mathrm{C}_{2} \mathrm{~T}_{x}$ nanosheets (Fig. S13, ESI $\dagger$ ), which causes a more disordered stacking microstructure. The reaction time dependence of $\mathbf{P A}$ and $\mathrm{Ti}_{3} \mathrm{C}_{2} \mathrm{~T}_{x}$ on the separation performance was also studied. The prolonged reaction time favors the transport of water molecules, as proved by the water permeance increasing from 271.9 to $709.8 \mathrm{~L} \mathrm{~m}^{-2} \mathrm{~h}^{-1} \mathrm{bar}^{-1}$ with the rejection maintained as $\sim 99 \%$ (Fig. 3b). However, once the reaction time between $\mathbf{P A}$ and $\mathrm{Ti}_{3} \mathrm{C}_{2} \mathrm{~T}_{x}$ lasts more than $4 \mathrm{~h}$, aggregation and sedimentation occur and result in irregular laminate stacking of the resultant membrane, thus a compromised rejection of $94.5 \%$ for CR. Summarily, the concentration of PA is optimized at $0.4-1.0 \mathrm{wt} \%$, with the reaction time with $\mathrm{Ti}_{3} \mathrm{C}_{2} \mathrm{~T}_{x}$ nanosheets fixed at $4 \mathrm{~h}$. These parameters enable sufficient interaction of PA molecules with $\mathrm{Ti}_{3} \mathrm{C}_{2} \mathrm{~T}_{x}$ nanosheets and ensure significant interlayer opening, whilst circumventing the defect formation from the sedimentation of MXene laminates.
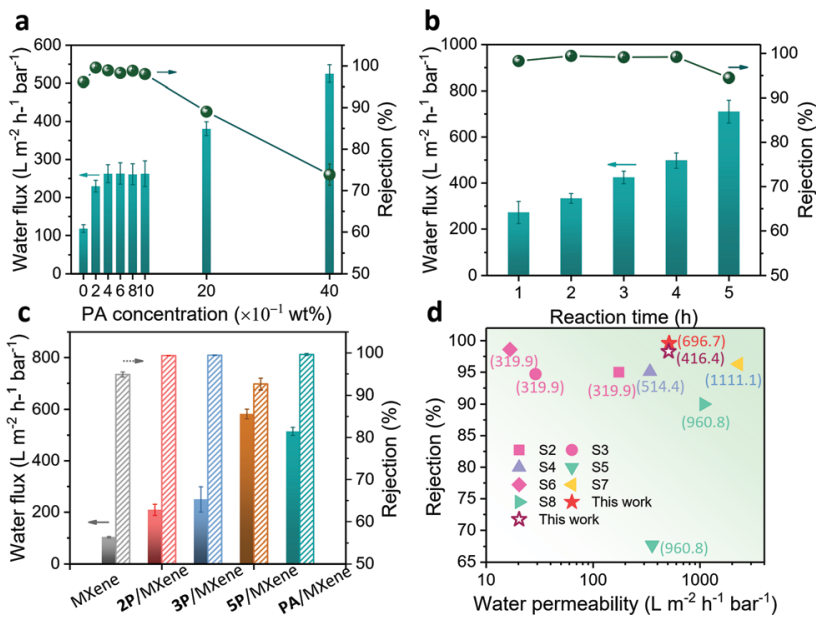

Fig. 3 (a) The effect of PA concentration and (b) reaction time on the separation performance of MXene membranes. Note that the reaction time for the PA concentration optimization was fixed at $1 \mathrm{~h}$. (c) Separation performance of pure and OPA modified MXene membranes for CR. (d) Comparison between the separation performances of the PA/MXene membrane and various previously reported membranes (marked with molecular weight of dye used in corresponding studies).

Then, molecule transport properties in various OPA modified MXene membranes were further investigated by filtering the aqueous solution containing organic dyes with different sizes and charges. Compared to the pure MXene membrane, the OPA modified MXene membranes with regulated nanochannels (2P/MXene, 3P/MXene and PA/MXene) perform much better in terms of both flux and rejection (Fig. 3c). Accordingly, the 5P/MXene membrane shows decreased rejection due to the sedimentation of 5P-modified $\mathrm{Ti}_{3} \mathrm{C}_{2} \mathrm{~T}_{x}$ nanosheets as discussed above. PA/MXene with the most enlarged and regulated interspace delivers a superior permeability of $\sim 510 \mathrm{~L} \mathrm{~m}^{-2} \mathrm{~h}^{-1} \mathrm{bar}^{-1}$, with remarkable rejection of 98.3\% for Eriochrome Black T (BT, MW of $461.38 \mathrm{~g} \mathrm{~mol}^{-1}$ ) and $99.7 \%$ for CR (Fig. 3c and Fig. S14, ESI $\dagger$ ). Note that the enhanced hydrophilicity of the OPA modified MXene membrane surface also contributes fast water transport, as the water contact angles of MXene membranes decrease after OPA modification (Fig. S15, ESI $\dagger$ ). Furthermore, the higher rejection of BT and CR for all membranes, in comparison to that of the positively-charged Methylene Blue (MB, MW of $319.85 \mathrm{~g} \mathrm{~mol}^{-1}$ ) and Rose Bengal (RB, MW of $1017.64 \mathrm{~g} \mathrm{~mol}^{-1}$ ) (Fig. 3c and Fig. S14, ESI $\dagger$ ), indicates that the rejection mechanism is synergistically dominated by size-exclusion effects and electrostatic interaction. ${ }^{25,29}$ As summarized in Fig. 3d and Table S3, ESI, $\dagger$ the PA/MXene membrane exhibits an exceptional water permeation and molecular sieving performance, highlighting the superiority of OPA-regulated transport nanochannels.

As an additional benefit, the interaction between OPA and MXene nanosheets endows them with better stability under varied $\mathrm{pH}$ conditions. After 24 days of immersion in different solutions, OPA modified MXene membranes maintained the original integrity, while the pure MXene membrane was oxidized completely. (Fig. 4a) More surprisingly, OPA modified MXene membranes present encouraging stability in acidic 

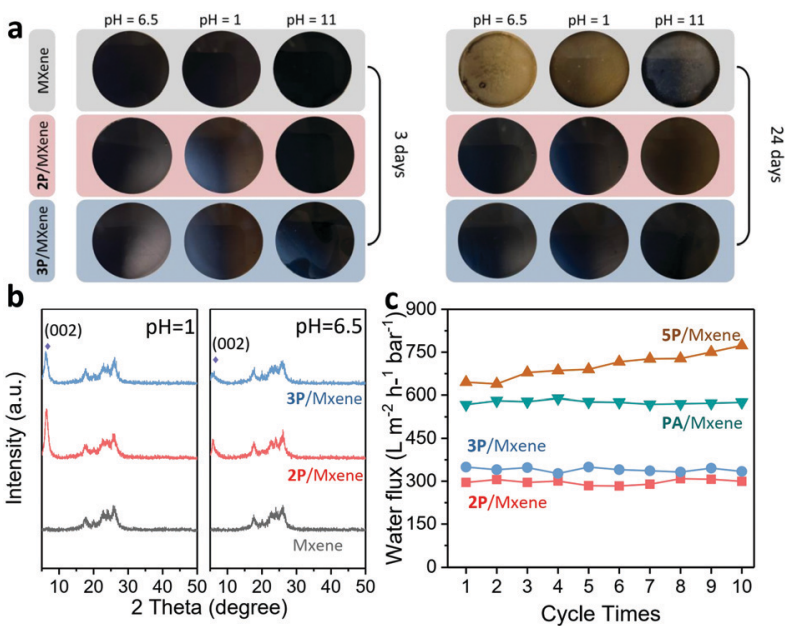

Fig. 4 (a) The stability of pure and OPA modified MXene membranes in natural, acid and alkaline solutions. (b) XRD patterns of the pure, 2P/MXene and 3P/MXene membrane after the solution immersion. (c) The variations of permeance with cycle times for pure and OPA modified MXene membranes.

$(\mathrm{pH}=1)$ and alkaline $(\mathrm{pH}=11)$ environments, demonstrating that OPA modified MXene membranes are fairly robust to tolerate harsh environments. XRD spectra were further collected to examine the regularity and integrity of the membranes' interlayer channels. Obviously, the peaks of the (002) plane for the pure MXene membrane disappear completely (Fig. 4b), suggesting the radical destruction and oxidation of MXene laminates. In contrast, OPA modified MXene membranes could still retain barely changed $d$-spacings (Fig. 4b) without oxidation. Such promising stability may be attributed to the capping effect of OPA molecules that blocks the water and oxygen attacking the metal sites of the nanosheets.

Besides the static test, the dynamic stability of the OPA modified membrane was also evaluated under long-term filtration of pure water. Excluding the 5P/MXene membrane, the OPA-modified MXene membranes show no significant decrease in permeance in a 10-cycle test (each cycle for 30 min filtration) (Fig. 4c), and the XRD results (Fig. S16, ESI $\dagger$ ) of the corresponding MXene also give a well-maintained $d$-spacing, showing their promising stability for practical applications.

In conclusion, we successfully regulated the nanochannels of MXene laminates via the intercalation of OPA molecules between adjacent nanosheets. Such membranes show five-fold increases in water permeance compared with the pure one. More importantly, owing to the strong interactions between OPA molecules and MXene nanosheets, these laminates possess relatively regular and stable stacking to diminish inevitable defects during the membrane fabrication. This contributes to the precise molecular sieving, and also the tolerance of membranes to harsh chemical environments. This study paves a way for the development of highly channel-regulated MXene membranes with superior selectivity and stability.
This work was supported by the Wallenberg Academy Fellow program (Grant KAW 2017.0166) in Sweden and the China Scholarship Council (202006160136).

\section{Conflicts of interest}

There are no conflicts to declare.

\section{Notes and references}

1 M. Naguib, M. Kurtoglu, V. Presser, J. Lu, J. Niu, M. Heon, L. Hultman, Y. Gogotsi and M. W. Barsoum, Adv. Mater., 2011, 23, 4248-4253.

2 M. R. Lukatskaya, O. Mashtalir, C. E. Ren, Y. Dall'Agnese, P. Rozier, P. L. Taberna, M. Naguib, P. Simon, M. W. Barsoum and Y. Gogotsi, Science, 2013, 341, 1502-1505.

3 A. Iqbal, P. Sambyal and C. M. Koo, Adv. Funct. Mater., 2020, 30, 2000883.

4 A. Liu, X. Liang, X. Ren, W. Guan, M. Gao, Y. Yang, Q. Yang, L. Gao, Y. Li and T. Ma, Adv. Funct. Mater., 2020, 30, 2003437.

5 O. Salim, K. A. Mahmoud, K. K. Pant and R. K. Joshi, Mater. Today Chem., 2019, 14, 100191.

6 K. Rasool, R. P. Pandey, P. A. Rasheed, S. Buczek, Y. Gogotsi and K. A. Mahmoud, Mater. Today, 2019, 30, 80-102.

7 H. E. Karahan, K. Goh, C. F. Zhang, E. Yang, C. Yildirim, C. Y. Chuah, M. G. Ahunbay, J. Lee, S. B. Tantekin-Ersolmaz, Y. Chen and T. H. Bae, Adv. Mater., 2020, 32, 1906697.

8 X. Xie, C. Chen, N. Zhang, Z. R. Tang, J. Jiang and Y. J. Xu, Nat. Sustainability, 2019, 2, 856-862.

9 G. Liu, W. Jin and N. Xu, Angew. Chem., Int. Ed., 2016, 55, 13384-13397.

10 J. Wang, Z. J. Zhang, J. N. Zhu, M. T. Tian, S. C. Zheng, F. D. Wang, X. D. Wang and L. Wang, Nat. Commun., 2020, 11, 3540.

11 G. Z. Liu, J. Shen, Y. F. Ji, Q. Liu, G. P. Liu, J. Yang and W. Q. Jin, J. Mater. Chem. A, 2019, 7, 12095-12104.

12 C. E. Ren, K. B. Hatzell, M. Alhabeb, Z. Ling, K. A. Mahmoud and Y. Gogotsi, J. Phys. Chem. Lett., 2015, 6, 4026-4031.

13 L. Ding, Y. Y. Wei, Y. J. Wang, H. B. Chen, J. Caro and H. H. Wang, Angew. Chem., Int. Ed., 2017, 56, 1825-1829.

14 S. Jiao, A. Zhou, M. Wu and H. Hu, Adv. Sci., 2019, 6, 1900529.

15 Y. Wu, H. Hu, C. Yuan, J. Song and M. Wu, Nano Energy, 2020, 74, 104812.

16 Y. D. Xing, G. Akonkwa, Z. Liu, H. Q. Ye and K. Han, ACS Appl. Nano Mater., 2020, 3, 1526-1534.

17 C. J. Zhang, S. Pinilla, N. Mcevoy, C. P. Cullen, B. Anasori, E. Long and S. H. Park, Chem. Mater., 2017, 29, 4848-4856.

18 V. Natu, M. Sokol, L. Verger and M. W. Barsoum, J. Phys. Chem. C, 2018, 122, 27745-27753.

19 S. Huang and V. N. Mochalin, Inorg. Chem., 2019, 58, 1958-1966.

20 D. Kim, T. Y. Ko, H. Kim, G. H. Lee, S. Cho and C. M. Koo, ACS Nano, 2019, 13, 13818-13828.

21 H. Chen, Y. Wen, Y. Qi, Q. Zhao, L. Qu and C. Li, Adv. Funct. Mater., 2019, 30, 1906996.

22 M. Alhabeb, K. Maleski, B. Anasori, P. Lelyukh, L. Clark, S. Sin and Y. Gogotsi, Chem. Mater., 2017, 29, 7633-7644.

23 Z. Li, H. Zhang, J. Han, Y. Chen, H. Lin and T. Yang, Adv. Mater., 2018, 30, 1706981.

24 Z. K. Li, Y. Wei, X. Gao, L. Ding, Z. Lu, J. Deng, X. Yang, J. Caro and H. Wang, Angew. Chem., Int. Ed., 2020, 59, 9751-9756.

25 M. Ding, H. Xu, W. Chen, Q. Kong, T. Lin, H. Tao, K. Zhang, Q. Liu, K. Zhang and Z. Xie, J. Mater. Chem. A, 2020, 8, 22666-22673.

26 Y. Wen, B. Wang, C. Huang, L. Wang and D. Hulicova-Jurcakova, Chemistry, 2015, 21, 80-85.

27 X. Wu, X. Cui, W. Wu, J. Wang, Y. Li and Z. Jiang, Angew. Chem., Int. Ed., 2019, 58, 18524-18529.

28 J. Ran, T. Pan, Y. Wu, C. Chu, P. Cui, P. Zhang, X. Ai, C. F. Fu, Z. Yang and T. Xu, Angew. Chem., Int. Ed., 2019, 58, 16463-16468.

29 L. Shen, M. Yi, L. Tian, F. Wang, C. Ding, S. Sun, A. Lu, L. Su and Y. Wang, J. Membr. Sci., 2019, 586, 84-97. 